\title{
PERFIL SOCIOECONÔMICO E OBSTÉTRICO DE PUÉRPERAS ASSISTIDAS EM UMA MATERNIDADE FILANTRÓPICA
}

Franciéle Marabotti Costa Leite ${ }^{1}$, Tatiane Kely Oliveira Barbosa², Jéssika Santiago Mota², Luciana de Cássia Nunes Nascimento $^{3}$, Maria Helena Costa Amorim ${ }^{4}$ Cândida Caniçali Primo ${ }^{5}$

RESUMO: Estudo descritivo, transversal, com abordagem quantitativa, cujo objetivo foi conhecer o perfil socioeconômico e obstétrico de puérperas assistidas em uma maternidade ao norte do Espírito Santo. Cerca de 54\% das mulheres estavam na faixa etária de 20 a 29 anos, 83,6\% eram casadas ou viviam com parceiro, 49,5\% declararam-se pardas, 32,8\% possuíam ensino médio completo, 49,8\% tinham renda de até um salário mínimo, 61,6\% pertenciam à classe C e 89,2\% foram atendidas pelo Sistema Único de Saúde. Verificou-se que 72,4\% tiveram de uma a duas gestações, 53,9\% com intervalo interpartal de 2 a 5 anos, $62,2 \%$ com parto cesárea, cerca de $60 \%$ realizaram sete consultas ou mais de pré-natal e $87 \%$ negaram histórico de abortamento. Conclui-se que o perfil de puérperas é semelhante ao encontrado em outros estudos e evidencia-se a necessidade do serviço estar atento à não realização do pré-natal entre puérperas jovens e alta prevalência de parto cesárea.

DESCRITORES: Período pós-parto; Cuidado pré-natal; Saúde da mulher; Enfermagem.

\section{SOCIO-ECONOMIC AND OBSTETRIC PROFILE OF PUERPERAE ASSISTED IN A PHILANTHROPIC MATERNITY UNIT}

ABSTRACT: The aim of this descriptive, cross-sectional and qualitative study was to investigate the socio-economic and obstetric profile of puerperae assisted in a maternity unit in the North of Espírito Santo. Approximately $54 \%$ of the women were in the age range 20 to 29 years old, $83.6 \%$ were married or lived with a partner, $49.5 \%$ stated that they were of mixed race, $32.8 \%$ had finished junior high school, $49.8 \%$ had an income of not more than one minimum salary, $61.6 \%$ belong to social class $\mathrm{C}$ and $89.2 \%$ were attended by the Unified Health System (SUS). It was ascertained that $72.4 \%$ had had one to two pregnancies, $53.9 \%$ with an interpartal interval of 2 to 5 years, $62.2 \%$ with Caesarean section, approximately $60 \%$ had had seven or more pre-natal consultations and $87 \%$ denied having a history of miscarriage. It is concluded that the profile of puerperae is similar to that found in other studies and the need is evidenced for the service to be attent to the non-undertaking of pre-natal consultations among young puerperae and the high prevalence of Caesarean births. DESCRIPTORS: Post-partum period; Pre-natal care; Women's health; Nursing.

\section{PERFIL SOCIOECONÓMICO Y OBSTÉTRICO DE PUÉRPERAS ASISTIDAS EN UNA MATERNIDAD FILANTRÓPICA}

RESUMEN: Estudio descriptivo, transversal, con abordaje cuantitativo, cuyo objetivo fue conocer el perfil socioeconómico y obstétrico de puérperas de una maternidad al norte de Espírito Santo. Aproximadamente 54\% de las mujeres estaban en la franja etaria entre 20 y 29 años, 83,6\% estaban casadas o vivían con pareja, 49,5\% se declararon pardas, 32,8\% completaron enseñanza media, 49,8\% tenían renta de hasta un sueldo mínimo, 61,6\% pertenecían a la clase C y 89,2\% fueron atendidas por el Sistema Único de Salud. Se ha verificado que 72,4\% tuvieron de una a dos gestaciones, 53,9\% con intervalo interpartal de 2 a 5 años, $62,2 \%$ con parto cesárea, aproximadamente $60 \%$ realizaron siete consultas o más de prenatal y $87 \%$ negaron histórico de aborto. Se concluye que el perfil de puérperas es semejante a lo encontrado en otros estudios, evidenciándose la necesidad del servicio poner atención a la no realización del prenatal entre puérperas jóvenes y alta prevalencia de parto cesáreo. DESCRIPTORES: Periodo posparto; Cuidado prenatal; Salud de la mujer; Enfermería.

\footnotetext{
${ }^{1}$ Enfermeira. Mestre em Saúde Coletiva. Doutoranda em Epidemiologia pela Universidade Federal de Pelotas - UFPel. Professora do Departamento de Enfermagem da Universidade Federal do Espírito Santo - UFES. Membro do Grupo de Pesquisa CUIDAR: Ensino e Pesquisa em Enfermagem. ${ }^{2}$ Acadêmica de Enfermagem da UFES.

${ }^{3}$ Enfermeira. Mestre em Saúde e Gestão do Trabalho. Professora do Departamento de Enfermagem da UFES. Membro do Grupo de Pesquisa CUIDAR: Ensino e Pesquisa em Enfermagem.

${ }^{4}$ Enfermeira. Doutora em Enfermagem. Professora do Curso de Graduação e do Programa de Pós-Graduação em Saúde Coletiva da UFES. Membro do Grupo de Pesquisa CUIDAR: Ensino e Pesquisa em Enfermagem.

${ }^{5}$ Enfermeira. Mestre em Saúde Coletiva. Professora do Curso de Graduação e do Mestrado Profissional em Enfermagem da UFES. Departamento de Enfermagem, UFES. Membro do Grupo de Pesquisa CUIDAR: Ensino e Pesquisa em Enfermagem.
}

Autor correspondente:

Franciéle Marabotti Costa Leite

Universidade Federal do Espírito Santo

Rua Lumberto Maciel de Azevedo, 405 - 29090-700 - Vitória-ES-Brasil

Email: emaildafran@ig.com.br
Recebido: 12/08/2012

Aprovado: 20/03/2013

Cogitare Enferm. 2013 Abr/Jun; 18(2):344-50 


\section{INTRODUÇÃO}

O puerpério inicia-se de uma à duas horas após a saída da placenta e tem seu término imprevisto. Didaticamente pode ser dividido em: imediato $\left(1^{\circ}\right.$ ao $10^{\mathrm{a}}$ dia), tardio $\left(11^{\circ}\right.$ ao $42^{\circ}$ dia), e remoto (a partir do $43^{\circ}$ dia $)^{(1)}$. Durante esse período os fenômenos involutivos atingem todo o organismo materno, ocorrendo alterações físicas, fisiológicas ${ }^{(2)}$ e também um momento de transformações psicológicas, visto as necessidades de novos aprendizados, de consolidação familiar e de laços afetivos ${ }^{(3)}$.

Nota-se que apesar de o puerpério ser um evento marcante na vida das mulheres é, geralmente, um período negligenciado, uma vez que as atenções se voltam muito mais para os bebês e há expectativa de que a mulher assuma o papel de mãe de imediato e sem dificuldades ${ }^{(4)}$. Assim, o puerpério revela-se como um acontecimento de grande transcendência no ciclo vital da mulher ${ }^{(5)}$.

Vale destacar nesta fase, a complexidade das mudanças provocadas pela vinda de um novo ser, sendo que não se restringem apenas às variáveis psicobiológicas, mas também a fatores socioeconômicos fundamentais nesse processo de mudança ${ }^{(6)}$. Verifica-se que o nível de instrução, o acesso ao serviço de saúde, a classe econômica, a vida conjugal, o meio em que está inserida, a carga cultural, a renda familiar, dentre outros, causam impactos de naturezas diversas. Esses podem variar, dependendo da história de vida de cada mulher, influenciando diretamente no período puerperal, como por exemplo, a associação entre o baixo nível socioeconômico e a depressão pós-parto ${ }^{(7)}$.

Diante do exposto, justifica-se a realização desse estudo que tem por objetivo conhecer o perfil socioeconômico e obstétrico de puérperas assistidas em uma maternidade filantrópica, ao norte do Estado do Espírito Santo, uma vez que tal pesquisa contribuirá para o direcionamento das ações realizadas pelos profissionais, subsidiando o cuidado e incrementando o ensino na área da saúde da mulher.

\section{MÉTODO}

Trata-se de estudo do tipo descritivo, com abordagem quantitativa. A população foi composta por puérperas assistidas em uma maternidade filantrópica e a amostra foi constituída por 323 mulheres. Os critérios de inclusão foram ser puérpera e estar internada na referida maternidade no período de coleta de dados. As mulheres foram convidadas a participar da pesquisa, orientadas quanto aos objetivos do estudo e assinaram do Termo de Consentimento Livre e Esclarecido. Foi realizado a entrevista de forma individual com registro em dois formulários, o primeiro continha as variáveis socioeconômicas (faixa etária, situação conjugal, raça/cor, escolaridade, renda familiar, classe econômica e tipo de atendimento) e variáveis obstétricas (número de gestação, tipo de parto, realização de pré-natal, número de consultas de pré-natal, abortamento e intervalo interpartal). O segundo instrumento utilizado, para identificar a classe econômica das participantes, foi o de Classificação Econômica Brasil da Associação Brasileira de Empresas de Pesquisa, que estima o poder de compra das pessoas e famílias urbanas, abandonando a pretensão de classificar a população em termos de classes sociais ${ }^{(8)}$. A coleta de dados foi realizada no período de julho a outubro de 2010.

Os programas Excel XP e Word XP foram usados para digitação dos formulários e criação do banco de dados para análise. A análise estatística, por meio do programa Social Package Statistical Science 17.0, apresentou os resultados na forma descritiva com a utilização de frequência relativa (\%) e absoluta (N). Este estudo foi aprovado pelo Comitê de Ética em Pesquisa do Centro Universitário do Norte do Espírito Santo, sob o parecer 044/2010.

\section{RESULTADOS}

Verifica-se que cerca de $54 \%$ das mulheres encontravam-se na faixa etária de 20 a 29 anos, 83,6\% eram casadas ou viviam junto com o parceiro, $49,5 \%$ declararam-se pardas e 32,8\% possuíam o ensino médio completo. Quanto à renda familiar, percebeu-se que 49,8\% possuíam renda de até um salário mínimo e a maioria (61,6\%) pertencia à classe $\mathrm{C}$. No que tange ao tipo de atendimento, 89,2\% foram atendidas pelo SUS (Tabela 1).

Ao verificar o perfil obstétrico (Tabela 2), constata-se que $72,4 \%$ das puérperas tiveram de uma a duas gestações, sendo que em $62,2 \%$ dos casos o parto foi do tipo cesárea, com intervalo interpartal de 2 a 5 anos (53,9\%). Quando questionadas sobre o número de consultas de pré-natal, cerca de $60 \%$ realizaram 7 consultas ou mais. Observa-se que grande parte (87\%) das participantes negou histórico de abortamento.

A maioria das participantes que realizaram o pré- 
-natal (54,2\%) encontra-se na faixa etária de 20 a 29 anos, refere-se como parda (49,8\%), pertence à classe C (62,1\%) e possui o ensino médio completo (33,2\%). Apesar do número reduzido de mulheres que não realizaram o pré-natal, $50 \%$ encontram-se na faixa etária de 13 a 19 anos, negras (75\%), pertencentes à classe econômica D $(50 \%)$ e com ensino fundamental incompleto (50,0\%).

Tabela 1 - Perfil socioeconômico de puérperas. Espírito Santo, 2010

\begin{tabular}{lcc}
\hline \multicolumn{1}{c}{ Variáveis } & $\mathbf{n}$ & $\mathbf{\%}$ \\
\hline Faixa etária & & \\
13 a 19 anos & 88 & 27,2 \\
20 a 29 anos & 174 & 53,9 \\
30 a 45 anos & 61 & 18,9 \\
Situação conjugal & & \\
Solteira & 51 & 15,8 \\
Casada/vive junto & 270 & 83,6 \\
Separada/divorciada & 02 & 0,6 \\
Raça / Cor & & \\
Branca & 51 & 15,8 \\
Negra & 92 & 28,5 \\
Parda & 160 & 49,5 \\
Amarela & 15 & 4,6 \\
Indígena & 05 & 1,5 \\
Escolaridade & & \\
Analfabeta & 03 & 0,9 \\
Fundamental incompleto & 94 & 29,1 \\
Fundamental Completo & 98 & 30,3 \\
Ensino médio completo & 106 & 32,8 \\
Superior incompleto & 08 & 2,5 \\
Superior completo & 14 & 4,3 \\
Renda familiar & & \\
Um salário mínimo ou menos & 161 & 49,8 \\
Dois salários mínimos & 96 & 29,7 \\
Acima de dois salários & 66 & 20,4 \\
Classe econômica & & \\
Classe A2 & 05 & 1,5 \\
Classe B1 & 05 & 1,5 \\
Classe B2 & 27 & 8,4 \\
Classe C & 199 & 61,6 \\
Classe D & 79 & 24,5 \\
Classe E & 08 & 2,5 \\
Tipo de atendimento & & \\
Sistema Único de Saúde (SUS) & 288 & 89,2 \\
Convênio & 23 & 7,1 \\
Particular & 12 & 3,7 \\
Total & $\mathbf{1 0 0}$ \\
\hline & & \\
\hline & & \\
& &
\end{tabular}

Tabela 2 - Perfil obstétrico de puérperas. Espírito Santo, 2010

\begin{tabular}{lcc}
\hline \multicolumn{1}{c}{ Variáveis } & n & \% \\
\hline Número de gestações & 234 & 72,4 \\
$1-2$ & 70 & 21,7 \\
$3-4$ & 19 & 5,9 \\
5 ou mais & & \\
Tipo de parto & 122 & 37,8 \\
Normal & 201 & 62,2 \\
Cesárea & & \\
Intervalo interpartal & 20 & 12,8 \\
Menor que dois anos & 84 & 53,9 \\
De 2 a 5 anos & 52 & 33,3 \\
Maior que 5 anos & & \\
Realizou pré-natal & 319 & 98,8 \\
Sim & 04 & 1,2 \\
Não & & \\
Número de consultas pré-natal & 04 & 1,2 \\
Nenhuma & 09 & 2,8 \\
1 a 2 consultas & 116 & 35,9 \\
3 a 6 consultas & 194 & 60,1 \\
7 consultas ou mais & & \\
Abortamento & 42 & 13 \\
Sim & 281 & 87 \\
Não & $\mathbf{3 2 3}$ & $\mathbf{1 0 0}$ \\
\hline Total & & \\
\hline
\end{tabular}

\section{DISCUSSÃO}

Identificou-se que a maioria das puérperas é predominantemente jovem, estando na faixa etária entre 20 e 29 anos, sendo assim, consideradas pertencentes ao grupo de menor risco obstétrico. Dados de estudo realizado em São Paulo, com o objetivo de caracterizar puérperas e recém-nascidos, revelaram semelhanças com a presente pesquisa, onde se demonstrou que $52 \%$ das mulheres tinham entre 20 e 29 anos. ${ }^{(9)}$ Entretanto, vale ressaltar em nosso estudo a porcentagem significativa $(27,2 \%)$ de puérperas adolescentes.

Quanto à situação conjugal, observa-se a prevalência de mulheres casadas, resultado similar ao de estudo, que constatou que $64,5 \%$ das puérperas eram casadas ou viviam com companheiro ${ }^{(10)}$. Em relação à raça/cor, evidencia-se no estudo o maior número de mulheres que relatam ser parda, dado que está em consonância com pesquisa que aponta que aproximadamente $69 \%$ das puérperas assistidas em uma maternidade referem ser da raça/cor parda ${ }^{(11)}$.

Apesar de um percentual de aproximadamente $33 \%$ de puérperas com ensino médio completo, 
Tabela 3 - Perfil socioeconômico de puérperas segundo a realização de pré-natal. Espírito Santo, 2010

\begin{tabular}{lcccccc}
\hline \multicolumn{1}{c}{ Variáveis } & \multicolumn{2}{c}{ Sim } & \multicolumn{2}{c}{ Realizou pré-natal } & \multicolumn{2}{c}{ Total } \\
& $\mathbf{n}$ & $\mathbf{0}$ & $\mathbf{n}$ & $\mathbf{0}$ & $\mathbf{n}$ & $\mathbf{\%}$ \\
\hline Faixa etária & & & & & & \\
13 a 19 anos & 86 & 27 & 2 & 50 & 88 & 27,2 \\
20 a 29 anos & 173 & 54,2 & 1 & 25 & 174 & 53,9 \\
30 a 45 anos & 60 & 18,8 & 1 & 25 & 61 & 18,9 \\
Raça / Cor & & & & & & \\
Branca & 51 & 16 & 0 & 0 & 51 & 15,8 \\
Negra & 89 & 27,9 & 3 & 75 & 92 & 28,5 \\
Parda & 159 & 49,8 & 1 & 25 & 160 & 49,5 \\
Amarela & 15 & 4,7 & 0 & 0 & 15 & 4,6 \\
Indígena & 5 & 1,6 & 0 & 0 & 05 & 1,5 \\
Classe econômica & & & & & & \\
Classe A2 & 5 & 1,6 & 0 & 0 & 05 & 1,5 \\
Classe B1 & 5 & 1,6 & 0 & 0 & 05 & 1,5 \\
Classe B2 & 27 & 8,5 & 0 & 0 & 27 & 8,4 \\
Classe C & 198 & 62,1 & 1 & 25 & 199 & 61,6 \\
Classe D & 77 & 24,1 & 2 & 50 & 79 & 24,5 \\
Classe E & 7 & 2,2 & 1 & 25 & 08 & 2,5 \\
Escolaridade & & & & & & \\
Analfabeta & 3 & 0,9 & 0 & 0 & 03 & 0,9 \\
Fundamental incompleto & 92 & 28,8 & 2 & 50 & 94 & 29,1 \\
Fundamental completo & 43 & 13,5 & 1 & 25 & 44 & 13,6 \\
Ensino médio incompleto & 53 & 16,6 & 1 & 25 & 54 & 16,7 \\
Ensino médio completo & 106 & 33,2 & 0 & 0 & 106 & 32,8 \\
Superior incompleto & 8 & 2,5 & 0 & 0 & 08 & 2,5 \\
Superior completo & 14 & 4,4 & 0 & 0 & 14 & 4,3 \\
Total & $\mathbf{3 1 9}$ & $\mathbf{1 0 0}$ & $\mathbf{4}$ & $\mathbf{1 0 0}$ & $\mathbf{3 2 3}$ & $\mathbf{1 0 0}$ \\
\hline
\end{tabular}

ressalta-se a baixa escolaridade no grupo pesquisado, o que também é demonstrado por estudiosos da área, que revelam o maior percentual $(29,6 \%)$ de mulheres com ensino fundamental completo, seguido de $27,1 \%$ de mulheres com o ensino fundamental incompleto ${ }^{(12)}$. Outra pesquisa destaca que cerca de $22 \%$ das puérperas tinham 8 anos completos de estudo ${ }^{(13)}$.

O maior percentual de mulheres com renda familiar de até um salário mínimo também é evidenciado por estudo realizado em uma maternidade de Fortaleza-Ceará, onde $46,3 \%$ das puérperas entrevistadas possíam renda de até um salário mínimo ${ }^{(14)}$. Quanto à classe econômica, evidencia-se o predomínio de participantes pertencentes à classe $\mathrm{C}$, classe desfavorecida economicamente. Esse achado vai de encontro a estudo feito em uma maternidade pública que destaca o maior percentual de mulheres pertencentes à classe $\mathrm{D}(64,5 \%)^{(10)}$.

Nota-se que a maternidade em estudo, enquanto filantrópica, conveniada ao SUS, apresentou o maior número de puérperas atendidas por esse sistema. Há cerca de 12 anos, o governo federal criou o Programa de Humanização do Pré-Natal (PHPN) como objetivo de melhorar a cobertura, reduzir os índices de morbimortalidade materno-infantil e estabelecer diretrizes da atenção pré-natal e da assistência ao parto no SUS. Cerca de $80 \%$ dos municípios brasileiros, até o ano de 2004, já eram beneficiados pelo PHPN e dados do Ministério da Saúde mostram que a cobertura da assistência à gestação e ao parto vem aumentando em todas as regiões do país nos últimos anos ${ }^{(15)}$.

Em relação ao perfil obstétrico revelou-se, dentre as participantes deste estudo que a maioria teve uma a duas gestações. Estudo feito em maternidade pública, em Fortaleza-Ceará ${ }^{(16)}$ identificou maior percentual $(48,1 \%)$ de mulheres que passaram pela primeira e segunda gestação. A prevalência de puérperas submetidas ao parto cesárea vai de encontro ao achado por outros autores $^{(17)}$ que apontam que $26,4 \%$, das mulheres reali- 
zaram a cesariana. Ainda, vale enfatizar que o elevado percentual de cesárea identificado em nosso estudo, supera, em muito, a taxa recomendada pela Organização Mundial de Saúde, que é inferior a $15 \%{ }^{(1)}$. Em relação ao intervalo interpartal observa-se que a maioria relatou intervalo de dois a cinco anos. Achados similares foram encontrados em estudo realizado em uma maternidade em Teresina-Piauí( ${ }^{(18)}$, onde $56,2 \%$ do grupo apresentou o mesmo intervalo.

Vale ressaltar, em nossa pesquisa, a quase totalidade de puérperas que realizou pré-natal, sendo que a maioria delas participou de 7 consultas ou mais. Estudo $^{(14)}$ realizado sobre os antecedentes obstétricos e à gestação atual de puérperas constatou que $98, \%$ das puérperas tinham sido assistidas às consultas de pré-natal. Entretanto, este mesmo estudo demonstrou a realização de 7 consultas ou mais de pré-natal em um grupo menor de mulheres (44,9\%).

Os resultados mostram que a grande maioria das entrevistadas negou histórico de abortamento. Este achado é semelhante ao evidenciado por pesquisa feita com o objetivo de conhecer o perfil de puérperas atendidas em um hospital universitário do Rio Grande do Norte ${ }^{(17)}$ que mostrou que $14,2 \%$ das mulheres relataram ter vivenciado o abortamento.

$\mathrm{Na}$ atual pesquisa verifica-se a maior porcentagem de mulheres que realizou pré-natal na faixa etária entre 20 e 29 anos, referem-se pardas, pertencem à classe C e com o ensino médio completo. Estudo feito no município do Rio Grande, localizado na planície costeira sul do Estado do Rio Grande do Sul ${ }^{(19)}$, revela que $80,2 \%$ das puérperas estudadas tinham idade igual ou inferior a 29 anos, 69,9\% eram brancas e 50,6\% das pacientes relataram 9 ou mais anos de escolaridade.

Já entre as puérperas que não realizaram pré-natal verifica-se o predomínio da faixa de 13 a 19 anos. A gravidez na adolescência é um fenômeno complexo, associado a grande número de fatores, como econômicos, educacionais e comportamentais, precipitando problemas e desvantagens decorrentes da maternidade precoce. Assim, deve-se elaborar estratégias que visem à redução de comportamentos de risco ${ }^{(20)}$. Neste grupo verifica-se também o predomínio da raça/cor negra. Dados apontam que, seja por razões sociais ou de discriminação, as mulheres negras têm menor acesso aos serviços de saúde de boa qualidade, à atenção ginecológica e à assistência obstétrica, seja no pré-natal, parto ou puerpério ${ }^{(21)}$.

Um achado interessante no presente estudo é o predomínio de mulheres com ensino fundamental incompleto entre as que não realizaram pré-natal. Esse achado está em consonância com pesquisa que assinala que aproximadamente $52 \%$ das mulheres que não fizeram o pré-natal ou compareceram de uma a três consultas eram analfabetas, ou seja, a escolaridade materna esteve fortemente associada ao número de consultas no pré-natal ${ }^{(11)}$.

Vale destacar que as mulheres que não fizeram o pré-natal pertenciam à classe econômica $\mathrm{D}$. Diante desse achado vale lembrar que a universalidade no acesso aos serviços de saúde é condição fundamental para a equidade. No SUS, a universalidade é garantida pela gratuidade no uso de serviços, evitando-se que barreiras econômicas restrinjam o consumo ${ }^{(22)}$. Esses resultados apontam a necessidade do município construir ações estratégias que devem ser desenvolvidas pelos serviços a fim de garantir e expandir o acesso às consultas de pré-natal às populações mais vulneráveis a não realização desta prática. Nesse contexto, a atenção primária e, em especial, a equipe de saúde da família, tem um papel importante em aproximar a mulher, no período gravídico puerperal, do serviço por meio de ações com foco no início precoce da assistência pré-natal, realizar a busca ativa de gestantes na comunidade e a implantação e incentivo à participação em programas de planejamento familiar.

\section{CONCLUSÃO}

Podemos concluir que houve semelhança com o perfil de puérperas apresentado na literatura. Sugere-se, portanto, que a faixa etária, a raça/cor, a escolaridade e a classe econômica podem influenciar no acesso aos serviços de saúde e, neste contexto, é fundamental que o profissional de saúde esteja atento a fim de contribuir para o alcance da equidade, universalidade e integralidade para a consolidação do SUS e modificação da situação encontrada.

\section{REFERÊNCIAS}

1. Ministério da Saúde (BR). Parto, aborto e puerpério: assistência humanizada à mulher. Brasília: Secretaria de Políticas de Saúde; Área de Saúde da mulher; Ministério da Saúde; 2001.

2. Soares C, Varela VDJ. Assistência de enfermagem no puerpério em uma unidade de atenção básica: incentivando o autocuidado [monografia]. Florianópolis (SC): Universidade Federal de Santa Catarina; 2007. 
3. Ministério da Saúde (BR). Pré-natal e puerpério: atenção qualificada e humanizada - manual técnico. Série A. Normas e Manuais Técnicos. Série Direitos Sexuais e Direitos Reprodutivos - Caderno $n^{0} 5$. Brasília: Ministério da Saúde; 2006. p. 158.

4. Salim NR, Araújo NM, Gualda DMR. Corpo e sexualidade: a experiência de um grupo de puérperas. Rev. Latino-Am. Enfermagem. [Internet] 2010;18(4) [acesso em 13 mar 2011]. Disponível:http://dx.doi. org/10.1590/S0104-11692010000400011.

5. Merighi MAB, Gonçalves R, Rodrigues IG. Vivenciando o período puerperal: uma abordagem compreensiva da Fenomenologia Social. Rev Bras Enferm. [Internet] 2006;59(6) [acesso em 13 mar 2011]. Disponível: http:// dx. doi.org/10.1590/S0034-71672006000600010

6. Vido MB. Qualidade de vida na gestação [dissertação]. Guarulhos (SP): Universidade Guarulhos; 2006.

7. Moraes IGS, Pinheiro RT, Silva RA, Horta BL, Souza PLR, Faria AD. Prevalência da depressão pós-parto e fatores associados. Rev. Saúde Públ. [Internet] 2006;40(1) [acesso 13 mar 2011]. Disponível: http:// dx.doi.org/10.1590/S0034-89102006000100011

8. Associação Brasileira de Empresas de Pesquisa (ABEP). Critério de Classificação Econômica Brasil. Dados com base no levantamento Sócio Econômico 2009. [acesso em 10 nov 2010]. Disponível: http://www.abep.org/novo/ Content.aspx?ContentID=302

9. Abrão ACFV, Marton ES. Perfil das puérperas e recémnascidos atendido no centro de assistência e educação em enfermagem da Universidade Federal de São Paulo. Acta Paul. Enferm. 2001;14(2):66-73.

10. Leite FMC, Amorim MHC, Nunes GF, Soares MFS, Sabino NQ. Perfil sociodemográfico e obstétrico de puérperas internadas em uma maternidade de alto risco no município da Serra, ES. Rev. Bras. Pesq. Saúde. [Internet] 2009;11(1) [acesso em 13 mar 2011]. Disponível: http://periodicos.ufes.br/RBPS/article/ view/444

11. Primo CC, Amorim MHC, Castro DS. Perfil social e obstétrico das puérperas de uma maternidade. Rev. enferm. UERJ. [Internet] 2007;15(2) [acesso em 08 mar 2011]. Disponível: http://www.facenf.uerj.br/v15n2/ v15n2a02.pdf

12. Spindola T, Penna LHG, Progianti JM. Perfil epidemiológico de mulheres atendidas na consulta do pré-natal de um hospital universitário. Rev Esc Enferm
USP. [Internet] 2006;40(3) [acesso em 08 mar 2011]. Disponível: http://www.scielo.br/pdf/reeusp/v40n3/ v40n3a09.pdf

13. Meincke SMK, Oliveira MRP, Trigueiro DRSG, Carraro TE, Gondim ETC, Collet N. Perfil socioeconômico e demográfico de puérperas adolescentes. Cogitare enferm. [Internet] 2011;16(3) [acesso em 08 mar 2011]. Disponível: http://ojs.c3sl.ufpr.br/ojs2/index.php/ cogitare/article/view/21561/16234

14. Dodt RCM, Oriá MOB, Pinheiro AKB, Almeida PC, Ximenes LB. Perfil epidemiológico das puérperas assistidas em umalojamento conjunto. Rev. enferm. UERJ. [Internet] 2010;18(3) [acesso em 08 mar 2011]. Disponível: http://www.facenf.uerj.br/v18n3/v18n3a02.pdf

15. Ministério da Saúde (BR). Projeto de avaliação nacional do Programa de Humanização do Pré-natal e Nascimento (PHPN). Brasília: Ministério da Saúde; 2004.

16. Verissimo OMI, Andrade BMG, Bezerra Filho JG, Bezerra JP, Carvalho ORM, Gonçalves FRF. Puérperas com vírus humano da imunodeficiência positivo (HIV+) e as condições de nascimento de seus recém-nascidos. [Internet] 2012;28 [acesso em 12 out 2012]. Disponível: http://revistas.um.es/eglobal/article/ viewFile/138561/139721

17. Galvão MCB, Davin RNB. Perfil de puérperas internadas em um hospital. Rev. enferm UFPE. [Internet] 2011;7(6) [acesso em 08 mar 2011]. Disponível: http://www. ufpe.br/revistaenfermagem/index.php/revista/article/ viewFile/1548/pdf_603

18. Lima GSP, Sampaio HAC. Influência de fatores obstétricos, socioeconômicos e nutricionais da gestante sobre o peso do recém-nascido: estudo realizado em uma maternidade em Teresina Piauí. Rev. Bras. Saude Matern. Infant. [Internet] 2004;4(3) [acesso 08 mar 2011]. Disponível: http://dx.doi.org/10.1590/S151938292004000300005

19. Gonçalves CV, Duarte G, Costa JSD, Quintana SM, Marcolin AC. Perdas de oportunidades na prevenção do câncer de colo uterino durante o pré-natal. Ciênc. saúde colet. [Internet] 2011;16(5) [acesso em 05 mar 2011]. Disponível: http://www.scielo.br/pdf/csc/v16n5/ a20v16n5.pdf

20. Chalem E, Mitsuhiro SS, Ferri CP, Barros MCM, Guinsburg R, Laranjeira R. Gravidez na adolescência: perfil sócio-demográfico e comportamental de uma população da periferia de São Paulo, Brasil. Cad. 
Saúde Pública. [Internet] 2007;23(1) [acesso em 05 mar 2011]. Disponível: http://www.scielo.br/pdf/\%0D/csp/ v23n1/18.pdf

21. Ministério da Saúde (BR). Atenção a Saúde das Mulheres Negras: perspectiva da equidade no pacto nacional pela redução da mortalidade materna e neonatal. Série F. Comunicação e Educação em Saúde. Brasília: Ministério da Saúde; 2005. p. 20.

22. Travassos C. Equidade e o Sistema Único de Saúde: uma contribuição para debate. Cad. Saúde Pública. [Internet] 1997;13(2) [acesso em 05 mar 2011]. Disponível: http:// dx.doi.org/10.1590/S0102-311X1997000200024 